\title{
GMR
}

\section{Association between single nucleotide polymorphisms in $A K T 1$ and the risk of prostate cancer in the Chinese Han population}

\author{
J.M. Liu ${ }^{1 *}$, S.H. Cheng ${ }^{2 *}$, C. Xia ${ }^{3 *}$, T. Deng ${ }^{4}$, Y.C. Zhu ${ }^{1}$, X. Wei ${ }^{1}$ Z.L. Huang ${ }^{1}$, \\ B.H. Liao' ${ }^{1}$, D.Y. Luo ${ }^{1}$, Y.G. Zhang ${ }^{5}$, T. Jin ${ }^{1}$, K.J. Wang ${ }^{1}$, J. Huang ${ }^{6}$ and H. Li ${ }^{1}$ \\ ${ }^{1}$ Department of Urology, \\ Institute of Urology (Laboratory of Reconstructive Urology) West China Hospital, \\ Sichuan University, Chengdu, Sichuan, China \\ ${ }^{2}$ Department of Radiology, Peking Union Medical College Hospital; \\ Department of Radiology, West China Hospital, Sichuan University, \\ Chengdu, Sichuan, China \\ ${ }^{3}$ West China School of Medicine, Sichuan University, Chengdu, Sichuan, China \\ ${ }^{4}$ Department of Urology, Minimally Invasive Surgery Center, \\ The First Affiliated Hospital of Guangzhou Medical University, \\ Guangzhou, China \\ ${ }^{5}$ The Periodical Press of West China Hospital, Sichuan University, \\ Chengdu, Sichuan, China \\ ${ }^{6}$ West China Hospital, Sichuan University, Chengdu, Sichuan, China \\ *These authors contributed equally to this study. \\ Corresponding author: J. Huang \\ E-mail: huangjinscu@gmail.com \\ Genet. Mol. Res. 16 (1): gmr16019469 \\ Received October 24, 2016 \\ Accepted December 5, 2016 \\ Published March 30, 2017 \\ DOI http://dx.doi.org/10.4238/gmr16019469
}

Copyright (C) 2017 The Authors. This is an open-access article distributed under the terms of the Creative Commons Attribution ShareAlike (CC BY-SA) 4.0 License.

ABSTRACT. AKT1, also known as v-akt murine thymoma viral oncogene homolog 1, is involved in the regulation of cell-survival and anti-apoptotic activities, which may affect the pathogenesis of various

Genetics and Molecular Research 16 (1): gmr16019469 
cancers. However, the association between genetic variants of $A K T 1$ and the risk of developing prostate cancer has not been investigated before. This study investigated the associations between three polymorphisms (rs1130214, rs3730358, and rs2494732) in $A K T 1$ and the risk of development of prostate cancer in the Chinese Han population. Sequenom MassARRAY \& iPLEX technology were used to genotype these polymorphisms in 493 Chinese Han patients with prostate cancer and 309 age-matched healthy individuals. Compared to the CC genotype of the rs 3730358 polymorphism, the CT genotype of the same polymorphism was strongly associated with a decreased risk of prostate cancer $(\mathrm{OR}=0.617,95 \% \mathrm{CI}=0.390-0.976, \mathrm{P}=0.037)$. However, there was no significant difference between the allele frequency of the rs3730358 polymorphism and those of the other two polymorphisms ( $\mathrm{P}$ $>0.05)$. Moreover, no significant difference was found in the haplotype analysis $(\mathrm{P}>0.05)$. Our study found that the variant genotype CT of rs3730358 of $A K T 1$ was associated with a decreased risk of prostate cancer, which suggested that this polymorphism could play an important role in the development of the disease.

Key words: $A K T 1$; Prostate cancer; Single nucleotide polymorphism; Allele frequency

\section{INTRODUCTION}

Prostate cancer (PC) is one of the commonest male malignancies, which accounted for $27 \%$ of new cancer cases and 10\% of cancer-related deaths in 2014 (Society American Cancer, 2014). Additionally, PC is a highly heterogeneous disease with insufficient understanding of its pathogenesis. Genetic factors, as well as lifestyle, exposure to sunlight, alcoholism, and environmental factors are closely related to prostate carcinogenesis. However, the genetic factors that contribute towards the pathogenesis of PC are still unclear. Recently, several genetic variations have been implicated in increasing the risk of PC. For instance, previous studies have demonstrated that toll-like receptors (TLRs), specifically TLR subclass 2 (TLR2), TLR4, and TLR9, may contribute to PC pathogenesis by stimulating prostate epithelial cell proliferation in response to infectious stimuli (Kundu et al., 2008; Mandal et al., 2012). Additionally, Murphy et al. (2012) found an association of 8q24 variants with risk of PC in Nigerian and Cameroonian men.

AKT (protein kinase B) is a $58 \mathrm{kDa}$ serine-threonine kinase whose phosphorylated form (p-AKT) is highly active in many malignancies (Tokunaga et al., 2006; Shimizu et al., 2007; Rychahou et al., 2008). AKT1 (RAC-alpha serine-threonine protein kinase), a subtype of AKT, has been shown to play an important role in the regulation of cell survival and in anti-apoptotic activities (Ahmed et al., 1997; Cardone et al., 1998; Niquet and Wasterlain, 2004; Vandermoere et al., 2005), which may contribute to the development of several diseases, including cancer. Interestingly, $A K T 1$ is overexpressed in PC tissues and is related to the progression of $\mathrm{PC}$ and the recurrence of prostate-specific antigen (PSA) (Ayala et al., 2004; Le Page et al., 2006).

The differential expression of $A K T 1$, which includes changes in both protein levels and

Genetics and Molecular Research 16 (1): gmr16019469 
conformation, may be explained by the presence of single nucleotide polymorphisms (SNPs) within the gene. Recently, it has been reported that several SNPs in $A K T 1$ are associated with the risk of developing various cancers. For example, rs2494750 in $A K T 1$ showed significantly altered risk for PC-specific mortality (Karyadi et al., 2015). Polymorphisms rs3803300, rs 1130214, and rs2494732 in $A K T 1$ were significantly associated with the survival of patients in the early stage of non-small cell lung cancer (Kim et al., 2012), whereas polymorphisms rs 1130214 and rs 3803300 were related to susceptibility to oral squamous cell carcinoma (Wang et al., 2015). However, there is still no systematic research about the association between SNPs in $A K T 1$ and the risk of development of PC. Therefore, the aim of the present study was to investigate the relationship between $A K T 1$ SNPs and pathogenesis of PC in a cohort of Chinese Han individuals.

\section{MATERIAL AND METHODS}

\section{Selection of SNPs}

We selected tagging SNPs from a $5 \mathrm{~kb}$ region flanking the gene and within the gene body of $A K T 1$ by using the tagger algorithm. Tagging SNPs were identified by using a cutoff value of $\mathrm{R}^{2}=0.8$ and a minor allele frequency greater than 0.1 in the Chinese population based on the genotype data of the HapMap Project (www.hapmap.org) from the Centre d'Etude du Polymorphisme Humain. Other SNPs previously reported to be associated with the risk of developing PC were also included. A total of 5 SNPs (rs3803300, rs1130214, rs3730358, rs2498799, and rs2494732), including the haplotype-tagging SNPs and potential functional SNPs, were selected for genotyping. However, rs3803300 and rs2498799 had to be abandoned because of the lack of appropriate polymerase chain reaction (PCR) conditions. Correspondingly, polymorphisms rs1130214, rs3730358, and rs2494732 were selected for this study.

\section{Study subjects}

This study included a total of 493 patients with PC and 309 age-matched healthy male individuals (control). All patients with $\mathrm{PC}$ had been evaluated by prostate biopsy according to the Guidelines on Prostate Cancer of the European Association of Urology in the Department of Urology, West China Hospital, Sichuan University, from March 2008 to January 2015 (Heidenreich et al., 2011). The inclusion criteria for individuals in the control group were: 1) normal serum concentration of PSA, 2) normal digital rectal examination (DRE), 3) no family history of PC, and 4) absence of other tumors. The clinical data of PC patients was extracted, which included age, occupation, dietary structure, family history of PC, clinical stage of PC, and associated complications. One milliliter of peripheral blood was drawn from both the patients and the control individuals and stored at $-80^{\circ} \mathrm{C}$ prior to use. All the patients permitted the use of clinical information and blood samples for scientific research. The protocols were

approved by the ethics committees of West China Hospital and all the participants provided written informed consent.

\section{Genotyping}

DNA was isolated from peripheral blood using the QIAamp DNA extraction kit

Genetics and Molecular Research 16 (1): gmr16019469 
(Qiagen, Germantown, MD, USA) by following the manufacturer's instructions. The DNA concentration was adjusted to $50 \mathrm{ng} / \mu \mathrm{L}$ and stored at $-80^{\circ} \mathrm{C}$. Genotyping assay was conducted by iPLEX ${ }^{\mathrm{TM}}$ on the MassARRAY ${ }^{\circledR}$ platform of Sequenom (Sequenom, San Diego, Amercia). Primers for detecting the three SNPs were designed using the Genotyping Tools \& MassARRAY Assay Design software (Bo'ao, Beijing, China). Multiplex PCR technology was used for PCR amplification in a 384-well plate. After the PCR amplification, free dNTPs in the reaction were removed by treatment with shrimp alkaline phosphatase, following which, the PCR products were extended by a single base (single base extension or SBE) using the single base primer. The iPLEX assay coupled with matrix-assisted laser desorption/ionization-time of flight (MALDI-TOF) identified the different iPLEX products of varying molecular weights extended by different bases at the SNP sites. Finally, information of the three SNPs was obtained. Table 1 lists the three SNP primer sequences that were used for PCR amplification and the iPLEX assay.

\begin{tabular}{|c|c|c|c|c|c|c|}
\hline$\frac{\text { Gene name }}{A K T I}$ & \begin{tabular}{|l|l|} 
SNP ID \\
rs 130214
\end{tabular} & $\begin{array}{ll}\mathrm{MAF} \\
0.2402\end{array}$ & $\begin{array}{l}\text { Major/minor allele } \\
\mathrm{G} / \mathrm{T}\end{array}$ & 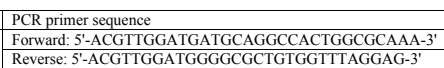 & \begin{tabular}{|l} 
iPLEX primer sequence \\
TCTCCCAGGAGGTTTTTG
\end{tabular} & \begin{tabular}{|l|l|l|l} 
Location \\
Intron
\end{tabular} \\
\hline AKTI & rs 33730358 & 0.1741 & $\mathrm{C} / \mathrm{T}$ & $\begin{array}{l}\text { Forvard: } 5 \text {-'ACGTGGAAGTCACAACTTCCTGGGGCG-3' } \\
\text { Reverse: }\end{array}$ & CCAAATCTGAATTCCGAGA & \begin{tabular}{|l|l} 
Intron \\
\end{tabular} \\
\hline AKTI & rs2494732 & 0.4259 & $\mathrm{C} / \mathrm{T}$ & 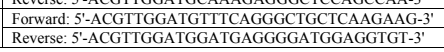 & GGGATGGAGGTGTAGCCTG & \begin{tabular}{|l|l|l} 
Intron \\
\end{tabular} \\
\hline
\end{tabular}

\section{Statistical analysis}

Statistical analysis was performed using the SPSS software (version 19.0; SPSS Inc., Chicago, IL, USA). A student's $t$-test was used to compare the differences in baseline data between the patients and control individuals, such as age. Pearson chi-square test was done to estimate the association between polymorphisms and the risk of developing PC. To evaluate the strength of this association, odds ratios (ORs) and 95\% confidence intervals (CIs) were calculated. Hardy-Weinberg equilibrium and haplotype analysis were tested by the SHEsis software (Shi and He, 2005; Li et al., 2009). All P values were two-sided, and P values $<0.05$ were considered as statistically significant.

\section{RESULTS}

\section{Demographic characteristics}

The demographic characteristics of patients with PC and control individuals are shown in Table 2. There was no significant difference in age distribution between patients and control individuals $(\mathrm{P}=0.34)$, whose median ages $(\mathrm{SD})$ were $69.94(9.11)$ and 70.41 (9.06) years, respectively. With respect to the clinical stages of the disease, the number of patients in grade II [187 (37.93\%)] and grade III [173 (35.09\%)] were higher than those in grade IV [122 $(24.75 \%)]$. Few patients were diagnosed to have grade I PC [11 $(2.23 \%)]$ in this study.

\section{Genotypic and allele frequencies of $A K T 1$ and the risk of PC}

The results obtained from the determination of the genotype and allele frequencies of

Genetics and Molecular Research 16 (1): gmr16019469 
AKT1 are listed in Table 3. The genotype frequencies of the polymorphisms rs 1130214 and rs2494732 in the control individuals were in Hardy-Weinberg equilibrium (rs1130214: $\chi^{2}=$ $\left.0.868, \mathrm{P}=0.351 ; \mathrm{rs} 2494732: \chi^{2}=0.051, \mathrm{P}=0.822\right)$. However, the genotype distribution of the polymorphism rs3730358 in control individuals was not in Hardy-Weinberg equilibrium $\left(\chi^{2}=\right.$ $8.546, \mathrm{P}=0.003)$. Interestingly, the genotype distribution of $\mathrm{rs} 3730358$ exhibited a significant difference between patients and controls. Compared with the $\mathrm{CC}$ genotype, the CT genotype was strongly associated with a decreased risk of $\mathrm{PC}(\mathrm{OR}=0.617 ; 95 \% \mathrm{CI}=0.390,0.976 ; \mathrm{P}$ $=0.037)$. However, the allele frequency of rs3730358 was not significantly different between the patients and the controls $(\mathrm{P}>0.05)$. For the remaining two SNPs, the genotype and allele frequencies did not differ significantly between the patient and the control groups $(\mathrm{P}>0.05)$.

Table 2. Demographic characteristics of the patients and control individuals.

\begin{tabular}{l|l|c|c|c}
\hline \multicolumn{2}{l|}{} & Cases $(\%)(\mathrm{N}=493)$ & Controls $(\mathrm{N}=309)$ & P value \\
\hline Mariables & & $69.94 \pm 9.11$ & $70.41 \pm 9.06$ & 0.34 \\
\hline Clinical stage & I & $11(2.23)$ & & \\
\hline Clinical stage & II & $187(37.93)$ & & \\
\hline Clinical stage & III & $173(35.09)$ & & \\
\hline Clinical stage & IV & $122(24.75)$ & & \\
\hline
\end{tabular}

Table 3. Association between selected SNP loci and the development of PC.

\begin{tabular}{|c|c|c|c|c|c|c|}
\hline Polymorphisms & Cases $(\mathrm{N}=493)(\%)$ & Controls $(\mathrm{N}=309)(\%)$ & $\chi^{2}$ & $\mathrm{P}$ & OR & $95 \% \mathrm{CI}$ \\
\hline \multicolumn{7}{|l|}{ rs1130214 } \\
\hline GG & $404(81.95)$ & $256(82.85)$ & & & 1.000 & \\
\hline GT & $87(17.65)$ & $49(15.86)$ & 0.363 & 0.547 & 0.889 & 0.606-1.304 \\
\hline TT & $2(0.40)$ & $4(1.29)$ & 0.947 & 0.330 & 3.156 & $0.574-17.357$ \\
\hline G & $895(90.77)$ & $561(90.78)$ & & & 1.000 & \\
\hline $\mathrm{T}$ & $91(9.23)$ & $57(9.23)$ & 0.363 & 0.547 & 0.889 & $0.606-1.304$ \\
\hline \multicolumn{7}{|l|}{ rs3730358 } \\
\hline $\mathrm{CC}$ & $417(84.58)$ & $276(89.32)$ & & & 1.000 & \\
\hline CT & $71(14.40)$ & $29(9.39)$ & 4.328 & 0.037 & 0.617 & $0.390-0.976$ \\
\hline TT & $5(1.02)$ & $4(1.29)$ & 0.079 & 0.779 & 1.209 & $0.322-4.541$ \\
\hline $\mathrm{C}$ & $905(91.78)$ & $581(58.92)$ & & & 1.000 & \\
\hline $\mathrm{T}$ & $81(8.22)$ & $37(41.08)$ & 2.767 & 0.096 & 0.712 & $0.476-1.064$ \\
\hline \multicolumn{7}{|l|}{ rs2494732 } \\
\hline $\mathrm{CC}$ & $271(54.97)$ & $178(57.61)$ & & & 1.000 & \\
\hline $\mathrm{CT}$ & $189(38.34)$ & $114(36.89)$ & 0.311 & 0.577 & 0.918 & $0.681-1.239$ \\
\hline TT & $33(6.69)$ & $17(5.50)$ & 0.602 & 0.438 & 0.784 & $0.424-1.451$ \\
\hline $\mathrm{C}$ & $731(74.14)$ & $470(76.05)$ & & & 1.000 & \\
\hline $\mathrm{T}$ & $255(25.86)$ & $148(23.95)$ & 0.740 & 0.390 & 0.903 & $0.715-1.140$ \\
\hline
\end{tabular}

$\mathrm{OR}=$ odds ratio $; 95 \% \mathrm{CI}=95 \%$ confidence interval.

\section{Haplotype analysis}

The association of $A K T 1$ SNPs with PC was further analyzed with the Haploview program. Frequencies of these haplotypes are listed in Table 4. The sequence of the SNP loci presented in Table 4 is in the order of rs1130214, rs3730358, and rs2494732. The frequencies of the 4 haplotypes (GCC, GCT, GTC, TCC) did not show significant difference between the patient and controls groups $(\mathrm{P}>0.05)$, while the remaining 4 haplotypes did not meet the requirements for the analysis. 
Table 4. Haplotype analysis of selected SNP loci and the development of PC.

\begin{tabular}{l|c|c|c|c|c}
\hline Haplotype & Cases (\%) & Controls (\%) & Chi $^{2}$ & P & OR (95\%CI) \\
\hline GCC & $586.30(59)$ & $385.82(62)$ & 0.978 & 0.323 & $0.900(0.729-1.110)$ \\
\hline GCT & $235.21(24)$ & $139.74(23)$ & 0.446 & 0.504 & $1.085(0.854-1.379)$ \\
\hline GTC & $64.26(7)$ & $29.71(5)$ & 2.135 & 0.144 & $1.394(0.891-2.180)$ \\
\hline GTT & $9.23(1)$ & $5.74(1)$ & - & - & - \\
\hline TCC & $76.16(8)$ & $52.92(9)$ & 0.304 & 0.582 & $0.902(0.625-1.301)$ \\
\hline TCT & $7.33(1)$ & $2.52(-)$ & - & - & - \\
\hline TTC & $4.28(-)$ & $1.55(-)$ & - & - & - \\
\hline TTT & $3.24(-)$ & $0.00(-)$ & - & - & - \\
\hline
\end{tabular}

The sequence of SNP loci is in the order of rs1130214, rs3730358, and rs2494732.

\section{DISCUSSION}

In this study, 802 individuals of the Chinese Han population were included to investigate the correlation between three SNPs (rs1130214, rs3730358, and rs2494732) of $A K T 1$ and the risk of developing PC. We found that variant genotype CT of rs3730358 was closely associated with a decreased susceptibility to PC. However, no relationship existed between rs 1130214 or rs2494732 and the risk of developing PC. Our findings suggested that rs 3730358 of $A K T 1$ could play a role in the development of PC and might be used as a potential predictive target for patients with $\mathrm{PC}$ in the Chinese Han population. To our knowledge, this is the first study to identify the correlation between SNPs of $A K T 1$ and the development of PC.

$A K T 1$ is located on chromosome 14q32.32 and consists of 14 exons. AKT1 can be activated by the integrin-linked kinase (ILK) with the assistance of the phosphatidylinositol 3-kinase (PI3K) and the phosphatidylinositol-dependent protein kinase (PDK) via the ILK/ PI3K/AKT pathway. Numerous studies have shown that the ILK/PI3K/AKT pathway is related to the regulation of cell-survival and anti-apoptotic activities through phosphorylation of Bad, Caspase-9, NF-кB, and c-Myc, by AKT1 (Ahmed et al., 1997; Cardone et al., 1998; Niquet and Wasterlain, 2004; Vandermoere et al., 2005). Thereafter, several studies were conducted to explore the association between AKT1 and cancer pathogenesis in recent years. SNPs are the most predominant genetic variations that are found in $A K T 1$. Certain $A K T 1$ SNPs were reported to be associated with cancer development. rs1130214 and rs2494732 were found to be nominally associated with the risk of developing PC in Caucasians and significantly associated with survival outcomes of patients in the early stages of non-small cell lung cancer in Korea (Kwon et al., 2011; Kim et al., 2012). rs3730358 has been shown to be involved in the modification of cancer risk in women with mutant $B R C A 1 / 2$ alleles (Ortiz-Cuaran et al., 2013). However, the association between SNPs on $A K T 1$ and the risk of developing PC in the Chinese population has not been explored so far.

We found significant difference for rs 3730358 between patients and control individuals. However, the relationship between rs 1130214 or rs 2494732 and the risk of developing PC did not exist in the Chinese Han population. Similarly, Zhang et al. (2014) also did not find any association between these two SNPs and the risk of developing nasopharyngeal carcinoma in the Chinese population through a case-control study consisting of 593 cases and 480 controls. Andreassen et al. (2013) obtained negative results upon investigating the association of seven $A K T 1$ SNPs (excluding rs1130214, rs3730358, and rs2494732) with the progression of testicular germ cell tumors in a large population of Norwegian-Swedish patients. rs3730358 and rs2494732 were also not related to the pathogenesis of oral squamous cell carcinoma in Chinese people (Wang et al., 2015). However, a population-based case-control study including

Genetics and Molecular Research 16 (1): gmr16019469 
1458 cases and 1351 controls conducted in Washington indicated that both rs1130214 and rs2494732 were considered to be related to PC risk in Caucasians (Kwon et al., 2011). The differences in the results could be attributed to the ethnic differences of the participants in our study and that by Kwon et al. More studies are required to confirm the relationship between these SNPs of $A K T 1$ and the risk of PC in different races.

The SNP rs3730358 is located in the intron of AKT1. Although the significance of SNPs in introns is not understood, several recent studies have shown the role of intron polymorphisms in linkage disequilibrium, with certain functional polymorphisms or genetic transcriptions associated with the risk of developing cancer or other diseases (Ortiz-Cuaran et al., 2013). Our study indicates that the variant allele $\mathrm{T}$ or the homozygous variant genotype (CT) of the intron SNP rs3730358 in AKT1 might also affect specific functional polymorphisms or transcription that are associated with susceptibility to PC.

Single nucleotide polymorphism includes single base conversions, transversions, insertions, and deletions. Therefore, we hypothesized that the formation of CT and TT genotypes involved different mechanisms. The SNP is located in an intron, and therefore, insertion or deletion of a single base may perturb the sequences of intron-exon splice junctions in patients harboring the CT genotype, which subsequently may affect splicing and translation of RNA, resulting in an altered susceptibility to PC. The TT genotype might arise due to spontaneous deamination of a methylated $\mathrm{C}$ to a $\mathrm{T}$, without altering the neighboring DNA sequence. In the latter case, splicing of the intron during RNA maturation would not change the arrangement of codons, thus not affecting the susceptibility to PC. Another explanation for the lack of any relationship between the TT genotype and susceptibility to PC is that the TT genotype is scarce in the Chinese population and the small sample size of this study was insufficient to detect any significant difference. Research on large number of samples on a nation-wide multi-center scale may help to verify these results in the future.

AKT1 expression might be regulated by the intron variant of SNP rs 3730358 , which could consequently affect the level of the mRNA and the protein. In this study, we found that the genotype distribution of polymorphism rs3730358 in control individuals did not follow the Hardy-Weinberg equilibrium. However, several factors, including selection bias, population stratification, and genotyping errors, could influence the Hardy-Weinberg disequilibrium. A non-significant result pertaining to the Hardy-Weinberg equilibrium is equivalent to results that do not reject the equilibrium assumption. Therefore, definite conclusions about rs 3730358 and whether it follows the Hardy-Weinberg equilibrium are difficult to make. In addition, it is difficult to assume Hardy-Weinberg equilibrium in populations. Deviations from the HardyWeinberg equilibrium demonstrate that one or more of the assumptions are invalid, which may not influence the result of the final association analysis (Schaid and Jacobsen, 1999). Therefore, the development of PC might be influenced by these factors. However, until now the precise mechanism by which the intron variant SNP rs3730358 decreases the risk of PC is unclear. More studies focused on the function of this SNP are required to confirm our results.

A further analysis was performed to explore the impact of the haplotypes on susceptibility to PC. In the haplotype analysis, we found that the 8 haplotypes (GCC, GCT, GTC, TCC, GTT, TCT, TTC, and TTT) might not contribute to the risk of developing PC in the Chinese Han population (in the order of rs1130214, rs3730358, and rs2494732). However, several studies have demonstrated that the haplotype of the polymorphisms selected in this study were associated with susceptibility to various diseases, probably due to altered expression of $A K T 1$. Emamian et al. (2004) found that lower levels of AKT1 in

Genetics and Molecular Research 16 (1): gmr16019469 
EBV-transformed lymphocytes were related to the haplotypes of rs 1130214 and rs3730358 in American patients of northern European descent with schizophrenia.

However, this study has certain limitations. Firstly, patients and control individuals were recruited from different populations - the patients were admitted to the hospital for treatment of PC, whereas the control individuals visited the hospital for other examinations. As a result, there could be inevitable selection bias. In order to minimize this limitation, patients and control individuals were matched according to their age. Moreover, to overcome the impact of inadequate matching, data was carefully adjusted and stratified. Secondly, as a small number of patients with the low-risk genotype group were included in this study, additional studies with larger sample sizes should be performed to confirm our results. Thirdly, patients and control individuals recruited in this study were mainly from the southwest of China, which might not represent all the characteristics of the Chinese Han population. Therefore, further studies that investigate the association between polymorphisms in this gene and susceptibility to PC should recruit patients and control individuals from various districts of China.

In conclusion, our study found that the variant $A K T 1$ genotype CT of rs 3730358 was associated with a decreased risk of PC. Conversely, no correlation was found between polymorphisms rs 1130214 and rs 2494732 and susceptibility to PC. These results suggest that rs3730358 of AKT1 could play a role in the development of PC. However, further studies are required to confirm and replicate our findings, especially in multiple ethnic populations. Furthermore, research about the effect of additional AKT1 SNPs on PC and their mechanisms of action that affect $\mathrm{PC}$ progression are also required.

\section{Conflicts of interest}

The authors declare no conflict of interest.

\section{ACKNOWLEDGMENTS}

Research supported by Grant \#2014K158 from the Administration of Traditional Chinese Medicine of Sichuan Province (J.M. Liu), Grant \#2014SZ0031 from the Science and Technology Administration of Sichuan Province (J.M. Liu), Grant \#31170907 (K.J. Wang), \#31370951 (K.J. Wang), \#81470927 (K.J. Wang), and \#81300579 (K.J. Wang) from the National Natural Science Foundation of China, Grant\#2014SCU04B21 (K.J. Wang) from Fund for Distinguished Young Scholars of Sichuan University, Grant \#JH2014053 (K.J. Wang) from the Academic Leader Training Fund of the Sichuan Province, and Grant \#JH2015017 (K.J. Wang) from the Application-Oriented Foundation of Committee Organization Department of the Sichuan Provincial Party.

\section{REFERENCES}

Ahmed NN, Grimes HL, Bellacosa A, Chan TO, et al. (1997). Transduction of interleukin-2 antiapoptotic and proliferative signals via Akt protein kinase. Proc. Natl. Acad. Sci. USA 94: 3627-3632. http://dx.doi.org/10.1073/pnas.94.8.3627 Andreassen KE, Kristiansen W, Karlsson R, Aschim EL, et al. (2013). Genetic variation in AKT1, PTEN and the 8q24 locus, and the risk of testicular germ cell tumor. Hum. Reprod. 28: 1995-2002. http://dx.doi.org/10.1093/humrep/det127

Ayala G, Thompson T, Yang G, Frolov A, et al. (2004). High levels of phosphorylated form of Akt-1 in prostate cancer and non-neoplastic prostate tissues are strong predictors of biochemical recurrence. Clin. Cancer Res. 10: 6572-6578. http://dx.doi.org/10.1158/1078-0432.CCR-04-0477

Genetics and Molecular Research 16 (1): gmr16019469 
Cardone MH, Roy N, Stennicke HR, Salvesen GS, et al. (1998). Regulation of cell death protease caspase-9 by phosphorylation. Science 282: 1318-1321. http://dx.doi.org/10.1126/science.282.5392.1318

Emamian ES, Hall D, Birnbaum MJ, Karayiorgou M, et al. (2004). Convergent evidence for impaired AKT1-GSK3beta signaling in schizophrenia. Nat. Genet. 36: 131-137. http://dx.doi.org/10.1038/ng1296

Heidenreich A, Bellmunt J, Bolla M, Joniau S, et al.; European Association of Urology (2011). EAU guidelines on prostate cancer. Part 1: screening, diagnosis, and treatment of clinically localised disease. Eur. Urol. 59: 61-71. http://dx.doi. org/10.1016/j.eururo.2010.10.039

Karyadi DM, Zhao S, He Q, McIntosh L, et al. (2015). Confirmation of genetic variants associated with lethal prostate cancer in a cohort of men from hereditary prostate cancer families. Int. J. Cancer 136: 2166-2171. http://dx.doi. org/10.1002/ijc.29241

Kim MJ, Kang HG, Lee SY, Jeon HS, et al. (2012). AKT1 polymorphisms and survival of early stage non-small cell lung cancer. J. Surg. Oncol. 105: 167-174. http://dx.doi.org/10.1002/jso.22071

Kundu SD, Lee C, Billips BK, Habermacher GM, et al. (2008). The toll-like receptor pathway: a novel mechanism of infection-induced carcinogenesis of prostate epithelial cells. Prostate 68: 223-229. http://dx.doi.org/10.1002/ pros. 20710

Kwon EM, Salinas CA, Kolb S, Fu R, et al. (2011). Genetic polymorphisms in inflammation pathway genes and prostate cancer risk. Cancer Epidemiol. Biomarkers Prev. 20: 923-933. http://dx.doi.org/10.1158/1055-9965.EPI-10-0994

Le Page C, Koumakpayi IH, Alam-Fahmy M, Mes-Masson AM, et al. (2006). Expression and localisation of Akt-1, Akt2 and Akt-3 correlate with clinical outcome of prostate cancer patients. Br. J. Cancer 94: 1906-1912. http://dx.doi. org/10.1038/sj.bjc. 6603184

Li Z, Zhang Z, He Z, Tang W, et al. (2009). A partition-ligation-combination-subdivision EM algorithm for haplotype inference with multiallelic markers: update of the SHEsis (http://analysis.bio-x.cn). Cell Res. 19: 519-523. http:// dx.doi.org/10.1038/cr.2009.33

Mandal RK, George GP and Mittal RD (2012). Association of Toll-like receptor (TLR) 2, 3 and 9 genes polymorphism with prostate cancer risk in North Indian population. Mol. Biol. Rep. 39: 7263-7269. http://dx.doi.org/10.1007/ $\underline{\text { s11033-012-1556-5 }}$

Murphy AB, Ukoli F, Freeman V, Bennett F, et al. (2012). 8q24 risk alleles in West African and Caribbean men. Prostate 72: 1366-1373. http://dx.doi.org/10.1002/pros.22486

Niquet J and Wasterlain CG (2004). Bim, Bad, and Bax: a deadly combination in epileptic seizures. J. Clin. Invest. 113: 960-962. http://dx.doi.org/10.1172/JCI21478

Ortiz-Cuaran S, Cox D, Villar S, Friesen MD, et al. (2013). Association between TP53 R249S mutation and polymorphisms in TP53 intron 1 in hepatocellular carcinoma. Genes Chromosomes Cancer 52: 912-919. http://dx.doi.org/10.1002/ gcc. 22086

Rychahou PG, Kang J, Gulhati P, Doan HQ, et al. (2008). Akt2 overexpression plays a critical role in the establishment of colorectal cancer metastasis. Proc. Natl. Acad. Sci. USA 105: 20315-20320. http://dx.doi.org/10.1073/ pnas.0810715105

Schaid DJ and Jacobsen SJ (1999). Biased tests of association: comparisons of allele frequencies when departing from Hardy-Weinberg proportions. Am. J. Epidemiol. 149: 706-711. http://dx.doi.org/10.1093/oxfordjournals.aje.a009878

Shi YY and He L (2005). SHEsis, a powerful software platform for analyses of linkage disequilibrium, haplotype construction, and genetic association at polymorphism loci. Cell Res. 15: 97-98. http://dx.doi.org/10.1038/ sj.cr.7290272

Shimizu Y, Segawa T, Inoue T, Shiraishi T, et al. (2007). Increased Akt and phosphorylated Akt expression are associated with malignant biological features of prostate cancer in Japanese men. BJU Int. 100: 685-690. http://dx.doi. org/10.1111/j.1464-410X.2007.07014.X

Society American Cancer (2014). Cancer Facts and Figures 2014. American Cancer Society, Atlanta.

Tokunaga E, Kimura Y, Oki E, Ueda N, et al. (2006). Akt is frequently activated in HER2/neu-positive breast cancers and associated with poor prognosis among hormone-treated patients. Int. J. Cancer 118: 284-289. http://dx.doi. org $/ 10.1002 /$ ijc. 21358

Vandermoere F, El Yazidi-Belkoura I, Adriaenssens E, Lemoine J, et al. (2005). The antiapoptotic effect of fibroblast growth factor-2 is mediated through nuclear factor-kappaB activation induced via interaction between Akt and IkappaB kinase-beta in breast cancer cells. Oncogene 24: 5482-5491. http://dx.doi.org/10.1038/sj.onc.1208713

Wang Y, Lin L, Xu H, Li T, et al. (2015). Genetic variants in AKT1 gene were associated with risk and survival of OSCC in Chinese Han Population. J. Oral Pathol. Med. 44: 45-50. http://dx.doi.org/10.1111/jop.12211

Zhang X, Chen X, Zhai Y, Cui Y, et al. (2014). Combined effects of genetic variants of the PTEN, AKT1, MDM2 and p53 genes on the risk of nasopharyngeal carcinoma. PLoS One 9: e92135. http://dx.doi.org/10.1371/journal.pone.0092135

Genetics and Molecular Research 16 (1): gmr16019469 